\title{
MicroRNAs are significantly influenced by p53 and radiation in HCT116 human colon carcinoma cells
}

\author{
SANGSU SHIN ${ }^{1}$, HWA JUN CHA ${ }^{1}$, EUN-MEE LEE ${ }^{1}$, JIN HYUK JUNG ${ }^{1}$, SU-JAE LEE ${ }^{2}$, \\ IN-CHUL PARK $^{3}$, YOUNG-WOO JIN ${ }^{4}$ and SUNGKWAN AN ${ }^{1,5}$ \\ ${ }^{1}$ Functional Genoproteome Research Centre, Konkuk University, Seoul 143-701; ${ }^{2}$ Department of Chemistry, \\ Hanyang University, Seoul 133-791; ${ }^{3}$ Laboratory of Functional Genomics, Korea Institute of Radiological \\ and Medical Sciences, Seoul 139-706; ${ }^{4}$ Division of Radiation Effect Research, Radiation Health \\ Research Institute of KHNP, Seoul 132-703; ${ }^{5}$ LIFEnGENE, Inc., Seoul 143-701, Korea
}

Received December 19, 2008; Accepted February 26, 2009

DOI: 10.3892/ijo_00000295

\begin{abstract}
Ionizing radiation is genotoxic to the cell, and p53 is commonly considered to be a key regulator that controls gene expression responding to the genotoxity of radiation. The expression profiles of microRNAs (miRNAs), which are small non-coding RNAs regulating the translation of target mRNAs, were analyzed to determine whether any correlation exists between miRNA expression, radiation response, and/or p53. The miRNA profiles were analyzed by microarray containing 470 human miRNA probes in HCT116 human colon carcinoma cells and their p53-null derivative. Thirty-eight miRNAs among the 138 flagged human miRNAs were selected by fold-change analysis. The expression levels of these 38 miRNAs were changed more than two-fold, and a total of 12 miRNAs were significantly affected by $\mathrm{p} 53$, radiation, and the combination of both. All 12 miRNAs had expression patterns correlated to p53, while two miRNAs were affected by radiation or the combined action of radiation and $\mathrm{p} 53$. In bioinformatics studies, these miRNAs had p53-binding sites with scores higher than $85 \%$ in their upstream regions, and some of their target genes were found to be involved in genotoxic responses. In conclusion, we have identified miRNAs influenced significantly by p53 and/or radiation in the HCT116 human colon carcinoma cell line model, and these miRNAs may have important roles in the regulation of genes involved the cellular responses to radiation.
\end{abstract}

\section{Introduction}

p53 is a tumor suppressor protein triggered by stress signals such as radiation, oxidative free radicals, hypoxia, and heat/

Correspondence to: Dr Sungkwan An, Functional Genoproteome Research Centre, Konkuk University, 1 Hwayang-dong, Gwangjin-gu, Seoul 143-701, Korea

E-mail: ansfgrc@konkuk.ac.kr

Key words: microRNA, expression profiles, p53, radiation exposure, HCT116 cold shock (1). It is mediated by many regulator proteins and induces various down-stream events including cell cycle arrest, apoptosis, DNA repair, and cellular senescence (2-6). In these processes, p53 plays an important role as a transcription factor to regulate the expression of related genes. Also, the p53 could regulate the translation of mRNA transcripts by binding to them, or induce cell death by accumulating in the mitochondria $(7,8)$.

Among the stress signals activating the p53, ionizing radiation causes the DNA double-strand breaks (DSBs). This is followed by the phosphorylation and stabilization of p53 by activated ataxia-telangiectasia mutated (ATM), a nuclear protein kinase and main transducer of DSB signals $(9,10)$. The activated p53 selectively induces cell cycle arrest or apoptosis $(11,12)$. These serial processes are controlled by DNA damage checkpoint pathways which inhibit the cell cycle progression through the G1 and G2 phases and delay the cell cycle in the irradiated cells. There are many other key proteins involved such as cycline-dependent kinases (CDKs) and the CDC25 family of proteins (13). Since ionizing radiation is applied in many cancer therapies, it is very important to understand the effects of radiation on cells.

Recently it was reported that p53 directly transactivates a microRNA (miRNA), miR-34a, and this miRNA reprograms gene expression and promotes apoptosis $(14,15)$. miRNAs are single-stranded small RNAs ( $22 \mathrm{nt})$ known to be important post-transcriptional regulators (16-19). They commonly inhibit the translation of target mRNAs by binding to the 3'-UTRs and thereby control many biological processes such as embryo development, cell fate and survival, tumorization, and disease (20-22). Xi et al showed that p53 acts as a transcription factor in regulating miRNA expression (23). There were many up- or down-regulated miRNAs depending on the existence of $\mathrm{p} 53$.

In this study, we investigated whether miRNA expression profiles are associated with p53 and radiation. We expected that miRNA expression is affected by radiation and that p53 is involved in the regulation of miRNA expression for radiation response. Our results matched these expectations, and we also discovered that the expression of two specific miRNAs is influenced most by radiation or the combined action of radiation and $\mathrm{p} 53$. 


\section{Materials and methods}

Cell culture. The human colon carcinoma cell lines, HCT116 (wt-p53) and p53-null derivative (null-p53), were cultured in a McCoy's 5A medium containing $10 \%$ fetal bovine serum (FBS) and antibiotics at $37^{\circ} \mathrm{C}$ in a humidified chamber supplemented with $5 \% \mathrm{CO}_{2}$. One day before irradiating, $5 \times 10^{5}$ cells were seeded onto $60 \mathrm{~mm}$ culture dishes.

Irradiation and RNA preparation. Cells were cultured for $24 \mathrm{~h}$ prior to irradiation with $10 \mathrm{~Gy}$, using a Gammacell ${ }^{\circledR}$ 3000 Elan irradiator ${ }^{137} \mathrm{Cs} \gamma$-ray source; MDS Nordin, ON, Canada). The irradiated cells were maintained for $24 \mathrm{~h}$ before extracting total RNA. The total RNA was extracted with TRIzol $^{\circledR}$ reagent (Invitrogen, CA, USA) according to the manufacturer's protocol. For the microarray studies, the quality and concentration of the RNA samples were determined with an Agilent 2100 Bioanalyzer (Agilent Technologies, CA, USA) and an Ultrospec 3300 Pro UV/Visible Spectrophotometer (Amersham Biosciences, NJ, USA). The recommended RNA quality parameters for the microarray analysis are an OD 260/280 ratio in the range of 1.8-2.0, an OD 260/230 ratio $>1.8$, an $18 \mathrm{~s} / 28 \mathrm{~s}$ rRNA ratio in the range of $1.8-2.1$, and an RNA integrity number (RIN) of $>8.0$.

Microarray analysis of miRNA profiles. The human miRNA microarray version $1 \mathrm{kit}$ (Agilent Technologies) containing probes for 470 human and 64 viral miRNAs was adapted to analyze the miRNA expression profiles. Before hybridizing the miRNA to the microarray, $100 \mathrm{ng}$ of total RNA was dephosphorylated with calf intestine alkaline phosphatase (CIP) and denatured with DMSO and heat. The dephosphorylated RNA was labeled with pCp-Cy3 using T4 RNA ligase, and the labeled RNA was purified with a Micro Bio-Spin P-6 column (Bio-Rad Laboratories, CA, USA). The purified RNA was denatured and hybridized to the microarray probes at $55^{\circ} \mathrm{C}$ and $20 \mathrm{rpm}$ for $20 \mathrm{~h}$ in the Agilent Microarray Hybridization Chamber (Agilent Technologies). The microarray slide was then washed with wash buffers and scanned to obtain the microarray image. The numerical data for the miRNA profiles were extracted from the image with the Feature Extraction program (Agilent Technologies). These data were analyzed with GeneSpring GX software version 7.3 (Agilent Technologies).

Classification of miRNAs and statistical analysis. Among the total miRNAs probed on the microarray, 470 human miRNAs were selected for further analysis. Then, the miRNAs whose flags were present in at least one sample were filtered and applied to the fold-change analysis. The fold-change analysis was conducted by factors of 2 -fold between four groups, HCT116 (wt-p53) control, irradiated HCT116 (wt-p53), HCT116 (null-p53) control and irradiated HCT (null-p53). Excluding the miRNAs showing no changes, the residual miRNAs were tested by two-way ANOVA with two parameters, p53 and radiation, using the GeneSpring software. Significant difference was set at $\mathrm{P}<0.05$.

Analysis of p53 binding sites upstream of miRNAs. In total, $50 \mathrm{~kb}$ upstream sequences of miRNAs were differentially expressed regardless of p53 were obtained by using UCSC human BLAT search (http://genome.ucsc.edu/cgi-bin/hgBlat). The p53 binding sites of miRNA upstream sequences were predicted and scored using the p53MH program (24). The top three binding sites were selected and listed.

Target prediction of miRNAs. The miRNAs whose expression was altered by $\mathrm{p} 53$ or radiation were used for target gene prediction. Two prediction programs, TargetScan 4.2 in the Whitehead Institute for Biomedical Research website (http://www.targetscan.org/) and miRBase Targets Version 5 in the Enright Lab at the Wellcome Trust Sanger Institute website (http://microrna.sanger.ac.uk/targets/v5/), were used. All predicted target genes from both programs were aligned by their gene names, and the genes present in both predictions were identified and listed.

\section{Results and Discussion}

To identify the expression profiles of miRNAs changed by radiation and $\mathrm{p} 53$, the human colon carcinoma HCT116 cell line and its derivative containing no p53 gene were adapted to analyze the miRNA profiles. There are a total of 534 miRNA probes on the microarray and the profiles of 470 human miRNAs were chosen for further assay. We selected 138 miRNAs by filtering for flags to ensure that the data are of good quality. Their profiles are diagramed in Fig. 1.

From the 138 flagged miRNAs, we excluded the miRNAs with expression levels changed less than 2-fold by comparing four groups: untreated and irradiated HCT116 cells containing wt-p53 and untreated and irradiated null-p53 HCT116 cells. We determined expression level changes in the presence/ absence of radiation and/or p53 and kept miRNAs with changes greater than 2-fold. We removed 108 miRNAs from the flagged set and kept 36 miRNAs for further study. These are listed in Fig. 2A and their expression profiles are color coded as in Fig. 1. The miRNA distribution and overlap for all groups are shown in a Venn diagram in Fig. 2B. Many miRNAs were shown to respond to $\mathrm{p} 53$ rather than radiation, that is, 35 miRNAs were included in D0 and D10 which meant the different expressions of miRNAs between wt-p53 and null-p53 HCT116 cells at each radiation dose (0-10 Gy), whereas 13 miRNAs belonged to DW and DN which meant the miRNAs changed by radiation in wt-p53 or null-p53 HCT116 cells.

The final 36 miRNAs were analyzed by two-way ANOVA to identify a primary factor in changing their expression levels. Twelve miRNAs were identified that were significantly affected by p53, radiation or both p53 and radiation (Table I, $\mathrm{P}<0.05)$. There were 12 miRNAs affected by $\mathrm{p} 53$, one miRNA by radiation, and two miRNAs by the combination of $\mathrm{p} 53$ and radiation. Their numerical expression levels are detailed in Table II. The miR-548c was affected by p53, radiation and combination of p53 and radiation. The miR-202 was affected by $\mathrm{p} 53$ and $\mathrm{p} 53$ with radiation, but not radiation alone. In the HCT116 (wt-p53) data set, miR-202, -330, and -516-5p expression levels were decreased, whereas miR-34a, $-34 b$, and $-548 \mathrm{c}$ were increased approximately 2 -fold by radiation. In the HCT116 (null-p53), miR-330, -485, -516-5p, and -548c were increased about 2 -fold or more by radiation. Note that 


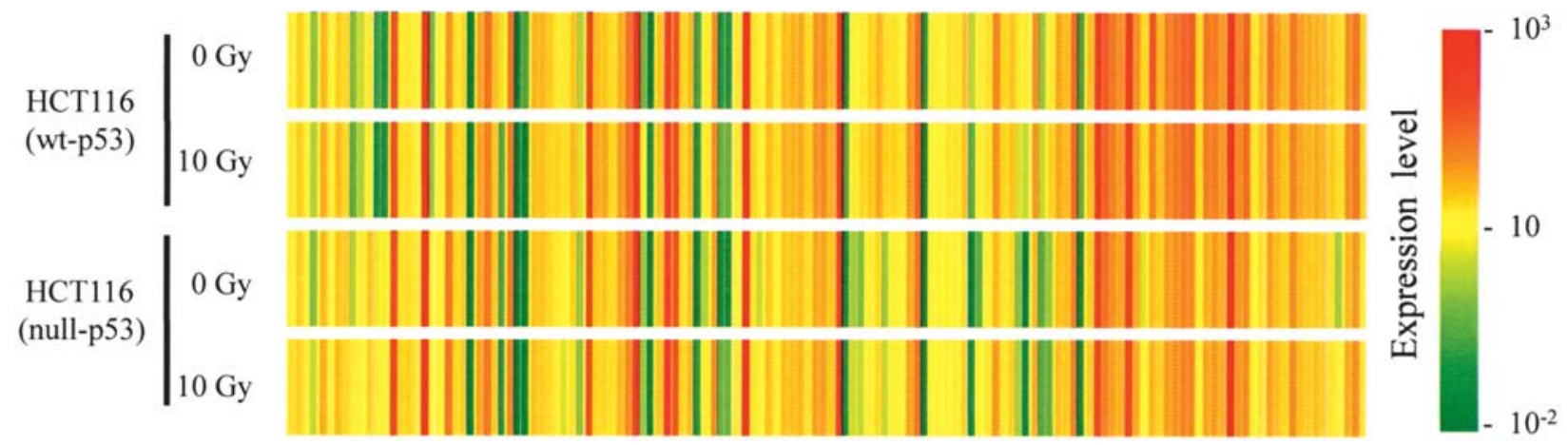

Figure 1. The expression profiles of miRNAs derived from microarray analysis. A total of 138 miRNAs were selected by flag filtering in the microarray data, and their expression levels are marked in color.

A

\section{HCT116 HCT116 \\ $\underline{\text { (wt-p53) (null-p53) }}$}

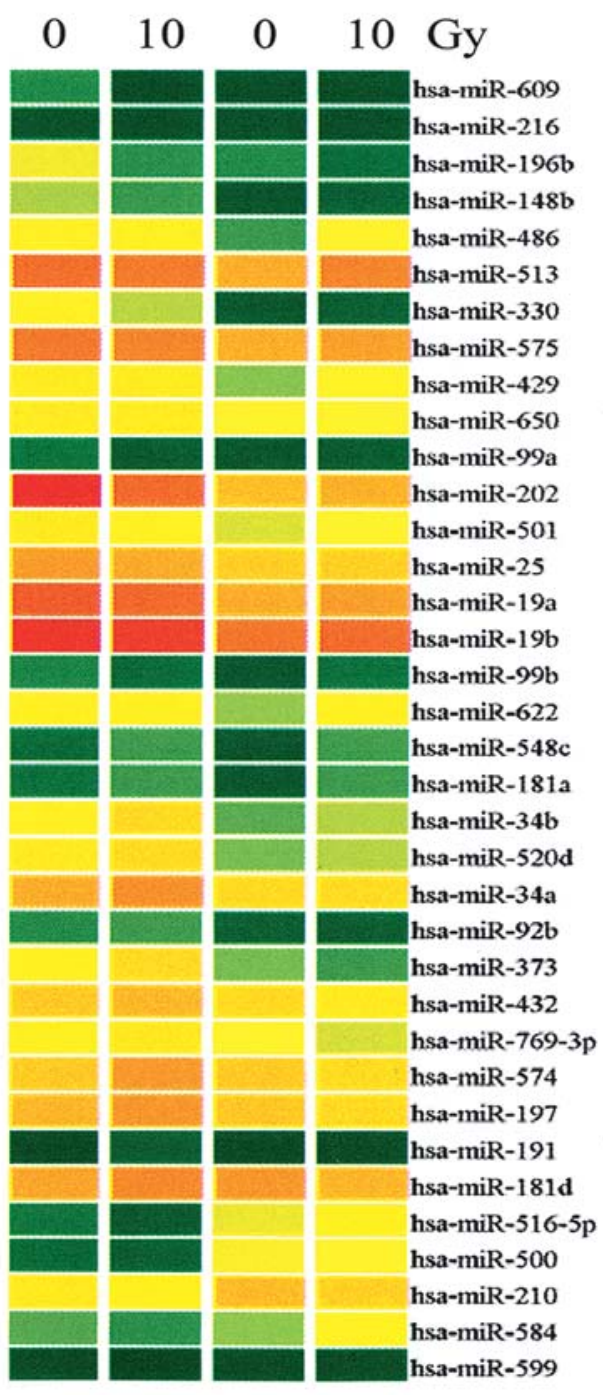

$\mathrm{B}$

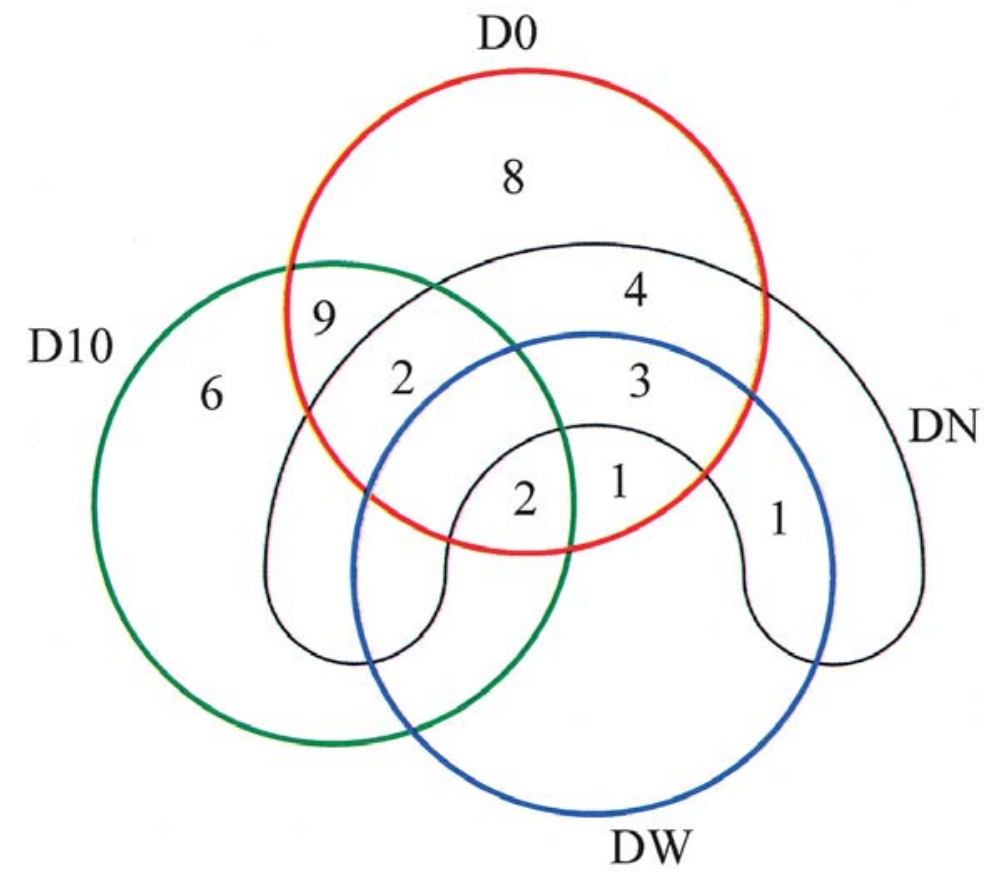

Figure 2. The miRNAs changed more than 2-fold between each group. For the flagged miRNAs, fold-change analysis was conducted, and the miRNAs showing more than 2-fold changes are listed with their expression levels by color (A). From this, total 36 miRNAs are described in a Venn diagram based on their distribution and overlap for all groups (B). The fold-change analyses were conducted between wt-p53 and null-p53 in 0 Gy (D0), wt-p53 and null-p53 in $10 \mathrm{~Gy}$ (D10), wt-p53 in 0 and $10 \mathrm{~Gy}$ (DW), and null-p53 in 0 and $10 \mathrm{~Gy}$ (DN). 
Table I. The list of miRNAs with expression levels changed by more than 2-fold by p53 and/or radiation.

\begin{tabular}{lll}
\hline Responding to & No. of miRNAs miRNA
\end{tabular}

p53 12

hsa-miR-19a, hsa-miR-34a, hsa-miR-34b, hsa-miR-202, hsa-miR-210, hsa-miR-330, hsa-miR-486, hsa-miR-500, hsa-miR-501, hsa-miR-516-5p, hsa-miR-548c, hsa-miR-575

Radiation $\quad 1 \quad$ hsa-miR-548c

Combination of p53 and radiation 2 hsa-miR-202, hsa-miR-548c

${ }^{\mathrm{a}} \mathrm{P}<0.05$ (error rate $=1.5$ genes) .

Table II. The average expression levels of miRNAs listed in Table I.

\begin{tabular}{|c|c|c|c|c|}
\hline \multirow[b]{2}{*}{ miRNA } & \multicolumn{2}{|c|}{$\begin{array}{l}\text { HCT116 } \\
\text { (wt-p53) }\end{array}$} & \multicolumn{2}{|c|}{$\begin{array}{l}\text { HCT116 } \\
\text { (null-p53) }\end{array}$} \\
\hline & 0 Gy & $10 \mathrm{~Gy}$ & 0 Gy & $10 \mathrm{~Gy}$ \\
\hline hsa-miR-19a & 190.7 & 159.8 & 71.7 & 76.7 \\
\hline hsa-miR-34a & 63.4 & 105.6 & 27.5 & 31.2 \\
\hline hsa-miR-34b & 18.9 & 30.2 & 6.2 & 8.4 \\
\hline hsa-miR-202 & 369.0 & 171.8 & 51.2 & 68.2 \\
\hline hsa-miR-210 & 19.6 & 17.2 & 48.2 & 44.5 \\
\hline hsa-miR-330 & 16.1 & 8.2 & 0.4 & 1.2 \\
\hline hsa-miR-486 & 17.0 & 16.2 & 5.2 & 10.8 \\
\hline hsa-miR-500 & 3.2 & 2.4 & 9.5 & 13.3 \\
\hline hsa-miR-501 & 20.2 & 14.7 & 9.0 & 10.0 \\
\hline hsa-miR-516-5p & 3.9 & 2.1 & 9.4 & 18.0 \\
\hline hsa-miR-548c & 2.6 & 5.4 & 0.6 & 5.5 \\
\hline hsa-miR-575 & 141.8 & 124.6 & 64.4 & 83.2 \\
\hline
\end{tabular}

after irradiation, miR-330 and -516-5p were down-regulated in the presence of $\mathrm{p} 53$, but up-regulated without $\mathrm{p} 53$.

Interestingly, the miR-34a, which is known as having the p53 binding site at about $30 \mathrm{~kb}$ upstream of the miRNA and being transactivated by p53 directly (14), is also shown to be affected by $\mathrm{p} 53$ in our results. In addition, miR-34b has been reported to have a p53 binding site about $3.6 \mathrm{~kb}$ upstream of the coding sequence (25), and it is affected by p53 in our study as well. The miR-34c, which is located beside the miR$34 \mathrm{~b}$ and transcribed by $\mathrm{p} 53$, did not show the significant fold change in our results. To address this apparent contradiction, we used a bioinformatics approach to examine whether the miRNAs affected by p53 had a direct upstream binding site for $\mathrm{p} 53$.

Twelve miRNAs significantly affected by p53 were analyzed with the p53MH program to identify p53-binding sites (24). Since the p53-binding site of miR-34a is located approximately $30 \mathrm{~kb}$ upstream from the miRNA sequence, we selected broader ranges of sequences about $50 \mathrm{~kb}$ upstream from pre-miRNA sequences. Among the many predicted binding sites, the top three binding sites were selected and listed in Table III. In this prediction, the scores of all predicted binding sites were $>85 \%$. Since the Mdm2 promoter with known p53-binding sites scored approximately 80\% (24), any sequence regions in this scoring range were considered strong candidates for having p53-binding sites. The scores of p53-binding sites of miR-34a and -34b reported previously were 100 and $93 \%$ in each. Interestingly, the miR-210 also showed scores of 100 and $93 \%$ at $23.4 \mathrm{~kb}$ and $9.5 \mathrm{~kb}$ upstream, respectively, indicating the likelihood of p53-binding sites.

Most of the miRNAs affected by p53 showed that expression levels are commonly down-regulated in the HCT116 (null-p53), but three miRNAs, miR-210, -500, and -516-5p, were up-regulated in the p53-null cells. They may have different pathways regulated by p53 from other miRNAs. For instance, the adenovirus E1B $19-\mathrm{kDa}$ protein and the cellular Bcl-2 participate in blocking p53-mediated apoptosis and transcriptional repression by p53 (26). Therefore, some other proteins could be involved in the regulation of p53mediated transcription of these miRNAs. In other words, those miRNAs could be repressed directly by binding of p53 to promoter regions in the regulation of genes such as $\mathrm{Bcl}-2$ and $\mathrm{Cdc} 25 \mathrm{C}(27,28)$. The miR-210 is located alone in the intron of AK123483 whose function is not known. The miR-210 is also known as being upregulated by hypoxia-inducible factor- $1 \alpha$, a transcription factor and independent prognostic factor in breast cancer (29). On the other hand, miR-500 and $-516-5 p$ are included in the miRNA clusters on chromosome Xp11.23 and 19q12.41, respectively. The exact mechanisms of transcriptional regulation for these miRNAs have to be confirmed by further studies.

Next, we analyzed the target genes of miRNAs by using two prediction programs, TargetScan 4.2 and miRBase Targets V5. Because they have different prediction algorisms, their predicted target genes were not exactly the same $(30,31)$, but some overlapped genes were selected and listed in Table IV. The numbers of target genes varied depending on the miRNAs. Many target genes were involved in regulation of 
Table III. The prediction of p53 binding sites upstream of miRNAs with expression altered by p53.

\begin{tabular}{|c|c|c|c|}
\hline \multirow[b]{2}{*}{ miRNA } & \multicolumn{3}{|r|}{ p53-binding site } \\
\hline & Distance $(\mathrm{kb})^{\mathrm{a}}$ & Score $(\% \max )^{\mathrm{b}}$ & Sequence \\
\hline \multirow[t]{3}{*}{ hsa-miR-19a } & 32.8 & 90.0 & CAACTAGTTT AACAGTAATTTTA TCACTTGCTT \\
\hline & 35.7 & 89.0 & ATACTTGTCA AGCAT AAACATGTTT \\
\hline & 32.9 & 85.1 & GAACAAGAAC TTA AGACTAGCTC \\
\hline \multirow[t]{3}{*}{ hsa-miR-202 } & 22.9 & 93.7 & CAGCATGCTC CC AGGCAAGCTG \\
\hline & 49.1 & 87.6 & AGACCAGCCT GGGCGACAGGACG AGACTAGTCT \\
\hline & 2.2 & 86.4 & GTGCATGCAC GTGTGTGTGGATGT GAGCATGCCT \\
\hline \multirow[t]{3}{*}{ hsa-miR-34a } & 30.4 & 100.0 & GGGCTTGCCT GGGCTTGTTC \\
\hline & 37.5 & 90.8 & AGGCATGCGC TAGCATGCCC \\
\hline & 0.8 & 87.6 & TGGCCTGCCC TCTGGATTTC AGACTTGCCT \\
\hline \multirow[t]{3}{*}{ hsa-miR-34b } & 14.3 & 94.0 & ATACTAGCTC TCACCTGA AGGCTTGCCC \\
\hline & 3.7 & 93.0 & AGTCTTGCTT CAACATAT AAACTAGTTT \\
\hline & 34.3 & 85.8 & CAACAAGCGA TTAT GAACATGCTT \\
\hline \multirow[t]{3}{*}{ hsa-miR-210 } & 23.4 & 100.0 & GAGCAAGCCC ACTTTGGA AAGCATGCTC \\
\hline & 9.5 & 93.0 & GAACAAGCCT GC AGCCAAGTCC \\
\hline & 29.8 & 87.5 & AGGCTTGCCA AGGGCACACA CCACATGCCC \\
\hline \multirow[t]{3}{*}{ hsa-miR-330 } & 16.2 & 89.2 & TAACTTGATC AGGG TGACATGTCT \\
\hline & 18.1 & 87.4 & GTGCATGCCT GTAGTCC CAGCTAGTTG \\
\hline & 45.5 & 86.5 & CACCATGTTG GCC AGGCTAGTCT \\
\hline \multirow[t]{3}{*}{ hsa-miR-486 } & 6.3 & 89.3 & CAGCATGCAC GTGCAAGGC CAGCATGCCC \\
\hline & 30.6 & 87.5 & GCACATGCCA CCACACC TGGCTAGTTT \\
\hline & 11.9 & 85.3 & TCTCTTGTTT CAGGCCATCAGAGG GGGCAAGCCC \\
\hline \multirow[t]{3}{*}{ hsa-miR-500 } & 30.6 & 89.2 & GGGCTAGCCA GCAG CAGCAAGCCG \\
\hline & 49.0 & 85.8 & ACTCTTGTTC CTTGATC AAGCATGTCC \\
\hline & 14.7 & 85.4 & GGGCCAGTCT GGCCCAGTA ACACAAGTCT \\
\hline \multirow[t]{3}{*}{ hsa-miR-501 } & 31.9 & 89.2 & GGGCTAGCCA GCAG CAGCAAGCCG \\
\hline & 0.5 & 85.5 & GGACAAGCTT ACACCTCTTTGA GAGCATGAGC \\
\hline & 16.0 & 85.4 & GGGCCAGTCT GGCCCAGTA ACACAAGTCT \\
\hline \multirow[t]{3}{*}{ hsa-miR-516-5p } & 17.2 & 90.6 & TGGCATGTCC AACCTAGCCC \\
\hline & 43.9 & 86.1 & CACCATGTTG GCC AGGCTAGTCT \\
\hline & 42.7 & 86.1 & CACCATGTTG GCC AGGCTAGTCT \\
\hline \multirow[t]{3}{*}{ hsa-miR-548c } & 49.7 & 98.2 & CAACTTGTCC AAGTCTATG AAGCTTGTTT \\
\hline & 36.8 & 90.7 & AGGCATGTGC CAGCAAGCCT \\
\hline & 11.1 & 89.0 & AAACAAGCTC CTATGCTAA ATACTAGCCA \\
\hline \multirow[t]{3}{*}{ hsa-miR-575 } & 25.2 & 91.2 & TGGCAAGTTT GCT GACCTTGTCC \\
\hline & 8.6 & 88.1 & GGTCTTGCCA TGTTGCCC AGGCTAGTCT \\
\hline & 8.6 & 86.4 & TGCCATGTTG CCC AGGCTAGTCT \\
\hline
\end{tabular}

aDistance of $\mathrm{p} 53$ binding site upstream from pre-miRNA sequence. ${ }^{\mathrm{b}}$ The calculated scores are presented as percentages. 
Table IV. The prediction of target genes of miRNAs.

\begin{tabular}{ll}
\hline miRNA & Predicted target genes \\
\hline hsa-miR-19a & ABCB7, ACBD5, ACTN1, ADSS, AFTPH, ANXA7, ARFGEF1, ARHGAP21, ARMC8, ASNA1, \\
& ATP10A, ATP2B2, B3GALNT2, BAMBI, BCL3, BTG1, C14orf129, C16orf14, C2orf42, CADM4, \\
& CAMTA1, CAST, CBX1, CC2D1A, CCDC126, CCM2, CCNL1, CCNT2, CCPG1, CD69, CHMP4B, \\
& CHST1, CNTFR, CXorf45, DGKG, DHX40, DLC1, DNAJA2, DOCK10, DSEL, E2F8, EDG1, ELK3, \\
& ENPP5, ETV5, FAM114A1, FAM43A, FAM46B, FAM80A, FAM83D, FASTK, FBXO8, FLNC, FMR1, \\
& FOXF2, GPR137B, GRK6, HIPK3, IMPDH1, ITPR1, IVNS1ABP, JAKMIP1, KCNA4, KIAA0367, \\
& KIAA1553, LIN9, LONRF1, LPP, LRP2, MAP3K12, MBD6, MEMO1, MID1IP1, MIER1, MON2, \\
& MPPED2, NME7, NPAS3, PAK6, PCDH10, PCDHA7, PFN1, PHF12, PLAA, PLXNC1, PNRC1, \\
& PRKAA1, PSAP, PSD, PTK2B, PTPN21, PTPRG, RAB21, RAB2B, RAB34, RAI2, RAP1A, RAPGEF4, \\
& RBMS1, REEP3, RFX1, RFX4, RHEBL1, RHOB, RNF111, RNF167, RUNX2, SCN1B, SEMA4C, \\
& SFMBT1, SGK, SLAIN1, SLC26A7, SLC9A6, SMARCA2, SNX17, SOCS1, SOX21, SPHK2, SPRYD3, \\
& ST3GAL5, ST8SIA3, TAF4, TBK1, TGIF1, TGM3, TNFRSF12A, TNIP1, TOR1B, USP6, VPS37A, \\
& VPS4B, WDR20, WNT1, WNT3, ZBTB10, ZDHHC7, ZFPM2, ZFYVE9, ZIC5, ZMYND11, ZNF217, \\
& ZNF533, ZNF704
\end{tabular}

hsa-miR-34a

ABCF3, ACSL4, ALDOA, ANK3, AP2S1, ASB1, AXL, B3GAT3, B4GALT2, BEST1, CACNB3, CAMSAP1, CBLN4, COPS7B, CRHR1, CUEDC1, DBC1, DDX17, DLL1, DPYSL4, EML5, EVI5L, FBXO10, FBXO30, FGD6, FNDC8, FOXG1B, FUT8, GALNT7, GDAP1L1, GPR22, GRK6, HCN3, JAKMIP1, JMJD1C, LGR4, LOXL3, LZTS2, MET, MPP2, MYRIP, NRIP3, NUMBL, PGM1, PNOC, PPP1R14D, RALGDS, RALGPS1, RPS6KA4, RRAS, SCN1A, SEMA4C, SGPP1, SHKBP1, SLCO3A1, SNX15, SYVN1, TAF5, TMEM109, TMEM55A, UHRF2, ZCCHC17, ZIC5, ZNF207, ZNF281

hsa-miR-34b

ARID1B, BRD4, C21orf66, FAM49B, FOXP1, PPP3CA

hsa-miR-202

ATXN7L2, BOLL, CAPN6, CASKIN1, CEP164, CHN2, CRTAP, CSMD1, DNAJB11, DNAJC1, EIF4EBP1, FAM96A, GPATCH3, GTPBP2, HAS2, HNRPC, IGF1R, IL10, KLHDC3, LRFN4, LRRN3, MLSTD1, MON2, MYRIP, NP, P4HA2, PANX2, PBX3, PKN2, PPARGC1B, PURG, PYGO2, RBM9, RNF13, SENP2, SLC25A4, SLC31A2, SLC37A4, SLCO5A1, SMAP1, SNX5, TRIM71, ZBTB10, ZSWIM4

hsa-miR-210

EFNA3, GPD1L, ISCU, LRRC62, SCRT1

hsa-miR-330

AFF4, AP2M1, APBB2, ARHGEF9, BCL9, BFSP1, C11orf57, C18orf34, C5orf15, CTDSPL2, DCK, EEF2K, FGFR1, FIGF, FNTB, FOXG1B, FRK, FRMPD2, GCG, GNAO1, GNL1, GPRASP2, GRIA3, GRID1, GRM5, GTF2H1, HNRPU, HOXD8, HTATIP2, ID2, IQCH, LYN, MRPL27, NFATC3, OTUD4, PCMT1, PCYT1B, PICALM, PPP1R1B, PRDM8, PTPN4, PURA, RAI2, RBM12, RLF, SEPT12, SFRS10, SKIL, SLITRK4, SOSTDC1, SOX21, SYT5, TARDBP, TCF12, TEAD1, TFEB, TRIM3, TRIP12, ZNF318, ZNF410, ZNF423

hsa-miR-486

BTAF1, CADM1, CCDC78, CNTNAP4, DYNLL1, FOXO1A, IRX5, MARK1, NCOA6, ODZ2, SFRS3, SHB, SNRPD1, SORCS1, SP5, ST5, STK4, TOB1, UNC5C

hsa-miR-500

B4GALNT3, PPP4R1, SORCS1

hsa-miR-501

ATP6V1H, CART1, ELAVL4, ERRFI1, GIF, HAS2, MAP2K1, MYB, MYCL1, NFIL3, PHF6, PNN, SFRS2, SFXN4, SLC35B3, TNNI2, UBAP1, VDAC2 
Table IV. Continued.

miRNA

Predicted target genes

hsa-miR-548c

ANKRD10, ANP32B, ARF6, ARHGAP15, ARID1A, BNC2, BRD1, BTBD14A, BTG1, C11orf58, C14orf103, C16orf70, C17orf85, C1QBP, C1QL3, C6orf111, CCNL1, CDH12, CDH9, CDK7, CHST11, CLIC1, CLIP1, CNTN1, COIL, CPNE4, CREB3L2, CYFIP1, DAZAP1, DDR1, DDX42, DUSP8, EBF1, EIF5B, ELAVL2, ENPP4, EPB41, FAM120C, FBXO30, FLT3, FMNL1, FOXP1, GATA6, GPBP1, GTF2H1, GTF2IRD1, H3F3A, HCN4, HDAC2, HMCN1, HMGB2, IGFBP2, KCNH5, KCNMA1, LRRN1, LUC7L2, MATR3, MIPOL1, MLL3, MLLT7, NAT12, NDFIP2, NOVA1, NUP153, OAF, ODC1, ODZ3, ORMDL3, PAN3, PCBP2, PCK1, PDE3A, PDE4D, PDIA4, PDLIM5, PLAU, PPP1CB, PPRC1, PRCC, PSMD3, PTPRD, PTPRZ1, RAB6IP1, RALGPS1, RANBP9, RASGEF1A, RBBP6, RBMS1, REV1, SC4MOL, SF1, SF3B1, SGCZ, SMARCE1, SPOCD1, SPOP, STAG2, STT3A, STX5, TBC1D9, TCBA1, TNKS1BP1, TOB1, TRIM63, TSPAN12, UBQLN2, UNC13C, UTX, WAPAL, YBX1, YIPF4, YIPF5, ZIC2, ZMYM2, ZNF281, ZNF638

hsa-miR-575

ACTL6A, ARHGAP21, ARID1B, BCL9,C17orf63, C1orf2, CDC27, CSMD3, DNAJB14, DNM1L, EPC2, FGF1, GCLC, HSD11B2, LRFN5, LRRN3, MAPKAPK3, NRP1, PITX3, RAB6IP1, RBM14, RGS3, RSBN1, SLC24A3, TMC7, TSC22D1, TSG101

biological processes (GO: 0050789) and metabolic processes (GO: 0008152) in gene ontology (GO). Other target genes were related to the stress response (GO: 0006950), cell cycle (GO: 0007049) and cell death (GO: 0008219), all processes that are involved in the response to genotoxic stresses.

In this study, we identified 12 miRNAs affected by p53, radiation, or both. All were affected by $\mathrm{p} 53$ and two miRNAs were regulated by the combined action of p53 and radiation. Considering that miRNA is an important post-transcriptional regulator, this result will provide some clues to understand the biological processes in the cellular response to radiation. Further studies will be conducted to find direct evidence that those miRNAs are affected by $\mathrm{p} 53$ and radiation, and regulate the gene.

\section{Acknowledgements}

We are grateful to all other members in our research group for their support and advice in regards to this study. This work was supported by the Ministry of Education, Science, and Technology (2007-03151) and the Ministry of Knowledge Economy (R-2006-1-043) of the Republic of Korea.

\section{References}

1. Levine AJ, Hu W and Feng Z: The p53 pathway: what questions remain to be explored? Cell Death Differ 13: 1027-1036, 2006.

2. Lowe SW, Schmitt EM, Smith SW, Osborne BA and Jacks T: p53 is required for radiation-induced apoptosis in mouse thymocytes. Nature 362: 847-849, 1993.

3. Brugarolas J, Chandrasekaran C, Gordon JI, Beach D, Jacks T and Hannon GJ: Radiation-induced cell cycle arrest compromised by p21 deficiency. Nature 377: 552-557, 1995.

4. Bond J, Haughton M, Blaydes J, Gire V, Wynford-Thomas D and Wyllie F: Evidence that transcriptional activation by $\mathrm{p} 53$ plays a direct role in the induction of cellular senescence. Oncogene 13: 2097-2104, 1996.
5. Smith ML, Ford JM, Hollander MC, et al: p53-mediated DNA repair responses to UV radiation: studies of mouse cells lacking p53, p21, and/or gadd45 genes. Mol Cell Biol 20: 3705-3714, 2000.

6. Nitta M, Okamura H, Aizawa S and Yamaizumi M: Heat shock induces transient $\mathrm{p} 53$-dependent cell cycle arrest at $\mathrm{G} 1 / \mathrm{S}$. Oncogene 15: 561-568, 1997.

7. Miller SJ, Suthiphongchai T, Zambetti GP and Ewen ME: p53 binds selectively to the 5' untranslated region of cdk4, an RNA element necessary and sufficient for transforming growth factor beta- and p53-mediated translational inhibition of cdk4. Mol Cell Biol 20: 8420-8431, 2000.

8. Marchenko ND, Zaika A and Mol UM: Death signal-induced localization of p53 protein to mitochondria. A potential role in apoptotic signaling. J Biol Chem 275: 16202-16212, 2000.

9. Banin S, Moyal L, Shieh S, et al: Enhanced phosphorylation of p53 by ATM in response to DNA damage. Science 281: 1674-1677, 1998

10. Canman CE, Lim DS, Cimprich KA, et al: Activation of the ATM kinase by ionizing radiation and phosphorylation of p53. Science 281: 1677-1679, 1998.

11. Kastan MB, Zhan Q, el-Deiry WS, et al: A mammalian cell cycle checkpoint pathway utilizing p53 and GADD45 is defective in ataxia-telangiectasia. Cell 71: 587-597, 1992.

12. Barlow C, Brown KD, Deng CX, Tagle DA and WynshawBoris A: ATM selectively regulates distinct p53-dependent cellcycle checkpoint and apoptotic pathways. Nat Genet 17: 453-456, 1997.

13. Iliakis G, Wang Y, Guan J and Wang H: DNA damage checkpoint control in cells exposed to ionizing radiation. Oncogene 22: 5834-5847, 2003.

14. Chang TC, Wentzel EA, Kent OA, et al: Transactivation of miR-34a by 53 broadly influences gene expression and promotes apoptosis. Mol Cell 26: 745-752, 2007.

15. Raver-Shapira N, Marciano E, Meiri E, et al: Transcriptional activation of miR-34a contributes to p53-mediated apoptosis. Mol Cell 26: 731-743, 2007.

16. Lee Y, Ahn C, Han C, et al: The nuclear RNase III Drosha initiates microRNA processing. Nature 425: 415-419, 2003.

17. Lagos-Quintana M, Rauhut R, Lendeckel W and Tuschl T: Identification of novel genes coding for small expressed RNAs. Science 294: 853-858, 2001.

18. Lau NC, Lim LP, Weinstein EG and Bartel DP: An abundant class of tiny RNAs with probable regulatory roles in Caenorhabditis elegans. Science 294: 858-862, 2001. 
19. Lee RC and Ambros V: An extensive class of small RNAs in Caenorhabditis elegans. Science 294: 862-864, 2001.

20. Lai EC: MicroRNAs are complementary to 3'-UTR motifs that mediate negative post-transcriptional regulation. Nat Genet 30 : 363-364, 2002.

21. Ambros V: The functions of animal microRNAs. Nature 431: 350-355, 2004

22. Alvarez-Garcia I and Miska EA: MicroRNA functions in animal development and human disease. Development 132: 4653-4662, 2005.

23. Xi Y, Shalgi R, Fodstad O, Pilpel Y and Ju J: Differentially regulated micro-RNAs and actively translated messenger RNA transcripts by tumor suppressor p53 in colon cancer. Clin Cancer Res 12: 2014-2024, 2006.

24. Hoh J, Jin S, Parrado T, Edington J, Levine AJ and Ott J: The p53MH algorithm and its application in detecting p53-responsive genes. Proc Natl Acad Sci USA 99: 8467-8472, 2002.

25. Corney DC, Flesken-Nikitin A, Godwin AK, Wang W and Nikitin AY: MicroRNA-34b and MicroRNA-34c are targets of p53 and cooperate in control of cell proliferation and adhesionindependent growth. Cancer Res 67: 8433-8438, 2007.
26. Shen $\mathrm{Y}$ and Shenk T: Relief of p53-mediated transcriptional repression by the adenovirus E1B $19-\mathrm{kDa}$ protein or the cellular Bcl-2 protein. Proc Natl Acad Sci USA 91: 8940-8944, 1994.

27. Wu Y, Mehew JW, Heckman CA, Arcinas M and Boxer LM: Negative regulation of bcl-2 expression by $\mathrm{p} 53$ in hematopoietic cells. Oncogene 20: 240-251, 2001.

28. St. Clair S, Giono L, Varmeh-Ziaie S, et al: DNA damageinduced downregulation of $\mathrm{Cdc} 25 \mathrm{C}$ is mediated by $\mathrm{p} 53$ via two independent mechanisms: one involves direct binding to the cdc25C promoter. Mol Cell 16: 725-736, 2004.

29. Camps C, Buffa FM, Colella S, et al: hsa-miR-210 is induced by hypoxia and is an independent prognostic factor in breast cancer. Clin Cancer Res 14: 1340-1348, 2008.

30. Lewis BP, Shih IH, Jones-Rhoades MW, Bartel DP and Burge CB: Prediction of mammalian microRNA targets. Cell 115: 787-798, 2003.

31. Enright AJ, John B, Gaul U, Tuschl T, Sander C and Marks DS: MicroRNA targets in Drosophila. Genome Biol 5: R1, 2003. 